\title{
XAV939 inhibits the stemness and migration of neuroblastoma cancer stem cells via repression of tankyrase 1
}

\author{
XIAOHONG TIAN, WEIJIAN HOU, SHULING BAI, JUN FAN, HAO TONG and HE XU \\ Department of Tissue Engineering, College of Basic Medical Sciences, \\ China Medical University, Shenyang 110001, P.R. China
}

Received January 28, 2014; Accepted March 13, 2014

DOI: $10.3892 /$ ijo.2014.2406

\begin{abstract}
Neuroblastoma (NB) is the most common extracranial solid tumor in childhood. One fundamental issue regarding NB recurrence and metastasis is the maintenance of cancer stem cells (CSCs) stemness. Tankyrase 1 (TNKS1) is overexpressed in several types of cancers and in NB cell lines. XAV939 is a small molecule inhibitor of TNKS1 and can induce apoptosis of NB cells. In this study, we showed that the surface marker CD133 method was more suitable for isolating NB CSCs than the side-population method, and $60 \mu \mathrm{M}$ etoposide was optimal for enriching NB CSCs. The NB CSCs were demonstrated in juvenescence or stemness state by electron microscopy, which was in line with the characteristics of CSCs. Furthermore, we demonstrated that the expression of the CSCs marker CD133 and migration ability of CSCs decreased after XAV939 treatment or by RNAi-mediated knockdown of the TNKS1 gene. These findings suggest that XAV939 treatment or RNAi-TNKS1 inhibits the stemness and migration of NB CSCs via the repression of TNKS1, and TNKS1 may be a potential molecular target for eliminating NB CSCs by small molecule drugs.
\end{abstract}

\section{Introduction}

The term cancer stem cells (CSCs) was defined as the small percentage of cells within a solid tumor capable of unregulated self-renewal, leading to continued tumor growth, as well as of the generation of partially differentiated progenitor cells (1-4). Neuroblastoma (NB) is the most common extracranial solid tumor in children and accounts for $8-10 \%$ of childhood cancers. Most children with high-risk NB have poor prognosis because of its ability to regress spontaneously, transform, or therapy-resistant relapse (5). The membrane of CSCs highly

Correspondence to: Professor Shuling Bai, Department of Tissue Engineering, College of Basic Medical Sciences, China Medical University, North 2nd Road 92, Heping Ward, Shenyang 110001, P.R. China

E-mail: shuling_bai@126.com

Key words: XAV939, cancer stem cells, neuroblastoma cell line, stemness expressed ATP-binding cassette (ABC) family of membrane transport proteins and multidrug resistance protein (MDRP), which can transport drugs and toxic substances outside the cell, and are not sensitive to conventional chemotherapy drugs, which is the main cause of chemotherapy failure (6). CSCs are deemed to be the source of tumor recurrence and metastasis. Only a tumor entirely eliminated of CSCs, can be cured completely. The CSC theory provides a new perspective for cancer research, and has gradually become a hot topic and a mainstream trend (7). The isolation and purification of CSCs is the foundation for targeted cure by the combine application of small molecule drugs and chemotherapeutic drugs.

XAV939 is a kind of small molecule tankyrase (TNKS) inhibitor and synthetized using a chemical genetics approach. Huang et al (8) and Chen et al (9) have verified that XAV939 could inhibit the proliferation of colon cancer cells by blocking Wnt signaling through binding to TNKS catalytic poly-ADPribose polymerase (PARP) domain. Tankyrase 1 (TNKS1) is a member of the TNKS family and upregulated in a variety of cancers, including multiple myeloma, plasma cell leukemia, high-grade non-Hodgkin's lymphomas, breast cancer, colon cancer and bladder cancer (10-16). These studies suggested that TNKS1 played a role in tumor progression. Our previous studies proved that TNKS1 was overexpressed in NB cell lines and XAV939 could induce apoptosis of NB cells partly by inhibiting Wnt/ $\beta$-catenin signaling through TNKS1 (17). However, it has not been reported whether XAV939 also has effect on stemness of NB CSCs, and the involved mechanism that would contribute to targeted therapy.

In the present study, we isolated, enriched and identified NB CSCs from SH-SY5Y cells. Then we repressed TNKS1 by XAV939 treatment or RNAi method, and demonstrated the inhibition effect on the stemness and migration ability of NB CSCs. We speculate that XAV939 is able to inhibit the stemness and migration of NB via repression of TNKS1, and TNKS1 might be a potential target for eliminating NB CSCs.

\section{Materials and methods}

Cell culture and TNKS1 inhibitor. Human NB SH-SY5Y cells were obtained from the American Type Culture Collection (ATCC; Rockville, MD, USA). Cells were cultured in Dulbecco's modified Eagle's medium-F12 (DMEM-F12; Hyclone), with $10 \%$ fetal bovine serum (FBS; Gibco), $100 \mathrm{U} / \mathrm{ml}$ 
penicillin, and $100 \mu \mathrm{g} / \mathrm{ml}$ streptomycin (Sigma Chemical Co., St. Louis, MO, USA) and were grown in a $5 \% \mathrm{CO}_{2}$ incubator at $37^{\circ} \mathrm{C}$. The TNKS1 inhibitor XAV939 was purchased from Sigma-Aldrich.

The comparison of isolation methods for human NB CSCs. For side population (SP) method, $10^{6}$ cells were collected and incubated in pre-warmed DMEM-F12/2\% FBS containing freshly added Hoechst 33342 (5 $\mu \mathrm{g} / \mathrm{ml}$ final concentration) for $90 \mathrm{~min}$ at $37^{\circ} \mathrm{C}$ with intermittent mixing. In control group, cells were incubated with the Hoechst dye in the presence of verapamil $(50 \mu \mathrm{M})$. At the end of incubation, cells were placed on ice at once and resuspended in cold DMEM-F12/2\% FBS until fluorescence-activated cell sorting (FACS) analysis. Before analysis, cells were stained with PI $(2 \mu \mathrm{g} / \mathrm{ml})$. The Hoechst dye was excited with the UV laser at 351-364 nm and its fluorescence measured with a $515 \mathrm{~nm}$ SP filter (Hoechst blue) and a 608 EFLP optical filter (Hoechst red). The emission wavelengths were separated by a 540 DSP filter. For surface marker method, $10^{7} \mathrm{SH}-\mathrm{SY} 5 Y$ cells were collected and suspended in FcR blocking reagent (Miltenyi Biotec) with 0.5\% FBS in PBS and added with mouse anti-human CD133/2(293C3)-PE monoclonal antibody which was diluted at 1:11. In addition, mixed well and refrigerated for $10 \mathrm{~min}$ in the dark $\left(4-8^{\circ} \mathrm{C}\right)$. Then cells were washed by adding 1-2 $\mathrm{ml}$ buffer per $10^{7}$ cells and centrifuged at $300 \mathrm{x}$ g for $10 \mathrm{~min}$, supernatant was aspirated completely and the cell pellet resuspended in DMEM-F12 with $2 \%$ FBS for isolating CD133 ${ }^{+}$and $\mathrm{CD}^{2} 33^{-}$cells, respectively, by flow cytometry. CD133+ cells were considered as NB CSCs. Mouse IgG2b-phycoerythrin was used as an isotype control and was set up for each sample.

The enrichment of $N B$ CSCs by etoposide treatment. The SH-SY5Y cells were plated at a density of $10^{5} / \mathrm{ml}$ in 6 -well plates. Etoposide (V 4629, Sigma-Aldrich, dissolved in PBS) of 20,40 and $60 \mu \mathrm{M}$ were added two days after cell seeding respectively, maintained for $24 \mathrm{~h}$, and removed by substituting the culture medium with fresh medium without drug. Untreated (control) cells were also subjected to change of medium at the same incubation times. Then the ratios of CSCs were analyzed by flow cytometry and the group of highest ratio was chosen. CSCs were sorted and cultured with DMEM/F12 medium contained $40 \mathrm{ng} / \mathrm{ml} \mathrm{bFGF}, 20 \mathrm{ng} / \mathrm{ml}$ EGF as well as $2 \%$ B27. This kind of medium contributes to prevent the differentiation of CSCs and maintain its characteristic.

Electron microscopy. The isolated $\mathrm{CD} 133^{+}$and $\mathrm{CD}^{-} 3^{-}$cells were cultured with different condition respectively. Some of them were cultured on coverslip for the observation by scanning electron microscope (SEM). When cells were at $60-70 \%$ confluence, the culture medium was discarded and cells were fixed in cold mixture of osmium tetroxide and glutaraldehyde for 1-3 h. Then samples were dehydrated in graded ethanol series, replaced with isoamyl acetate, dried at critical point, coated with gold on surface, and observed by JSM-T300 SEM. Another portion of cells were cultured in 6-well plates. The monolayer cells were collected and fixed with $2.5 \%$ glutaraldehyde for $2 \mathrm{~h}$. The cells were fixed with $1 \%$ osmium tetroxide for 2-3 h, dehydrated with graded ethanol, replaced with acetone, pelleted and embedded in $3 \%$ agar. The agarized pellet was cut into 1-mm slices and immersed in 3\% uranyl acetate-lead citrate for double staining, and viewed with a transmission electron microscope (TEM, JEM1200EX).

XAV939 treatment and RNAi. The isolated NB CSCs were cultured and proliferated for XAV939 treatment and RNAi. XAV939 was dissolved in dimethyl sulfoxide (DMSO, Sigma-Aldrich) and the final concentration was $1 \mu \mathrm{M}$ based on our previous study results (16). The control group was treated with DMSO only. The TNKS1 gene was knocked down by TNKS1-shRNA (shRNA group) while cells in control group were transfected with lentivirus-mediated scrambled-shRNA (SCR group). The XAV939 treatment, viral transfection and control groups were maintained for $72 \mathrm{~h}$. Then all groups were subjected to quantitative real-time RT-PCR (qRT-PCR) analysis, western blot analysis, immunofluorescence and migration assay.

QRT-PCR analysis. Total RNA was extracted using the RNeasy Mini kit (Qiagen, Valencia, CA, USA) and quantitated using NanoDrop 1000 (NanoDrop, Wilmington, DE, USA). Then the RNA was reverse transcribed into cDNA using PrimeScript $^{\circledR}$ RT reagent kit with gDNA Eraser (Takara). QRT-PCR was performed using SYBR ${ }^{\circledR}$ Premix Ex Taq ${ }^{\mathrm{TM}}$ II (Takara) on ABI 7500 Real-Time PCR system (Applied Biosystems). Sequences of the primers for CD133 were 5'-AGT GGCATCGTGCAAACCTG 3 (forward) and 5'-CTCCGAAT CCATTCGACGATA-3' (reverse). Sequences of the primers for $\beta$-actin were 5'-TGGCACCCAGCACAATGAA-3' (forward) and 5'-CTAAGTCATAGTCCGCCTAGAAGCA-3' (reverse). The PCR amplification was done at: $95^{\circ} \mathrm{C}$ for $30 \mathrm{sec} ; 95^{\circ} \mathrm{C}$ for $5 \mathrm{sec}, 60^{\circ} \mathrm{C}$ for $34 \mathrm{sec}$ for 40 cycles. The relative changes in gene expression data were analyzed by the $2-\Delta \Delta \mathrm{CT}$ method. $\beta$-actin was used as an internal control. Triplicates were run for each sample in three independent experiments.

Western blot analysis. The NB CSCs were lysed with RIPA buffer and protein concentration was determined by the Bradford method. Equal amounts of protein $(40 \mu \mathrm{g})$ were used for western blot analysis with primary antibodies to anti-CD133 (Bioss, bs-0209R) and anti- $\beta$-actin (Santa Cruz, sc-1616-R). Specific antibody binding was detected by horseradish peroxidase-conjugated goat anti-rabbit antibodies and visualized with ECL reagent (Santa Cruz) according to the manufacturer's protocol. Antibody to $\beta$-actin was used to evaluate protein loading in each lane.

Immunofluorescence. The NB CSCs were fixed with $4 \%$ paraformaldehyde for $10 \mathrm{~min}$ at room temperature, and permeabilized with $0.5 \%$ Triton X-100 for $15 \mathrm{~min}$. After blocking with $1 \%$ BSA for 30 min, cells were incubated with the primary antibody (CD133, Bioss, 1:200) for $2 \mathrm{~h}$ at $37^{\circ} \mathrm{C}$, then incubated with secondary fluorescein conjugated antibody (Bioss, 1:500) for $1 \mathrm{~h}$ at $37^{\circ} \mathrm{C}$ in the dark. To ensure specificity of results, negative controls with no primary antibody or no secondary antibody were used. The coverslips were incubated with Hoechst $33342(5 \mu \mathrm{g} / \mathrm{ml}$ for $10 \mathrm{~min})$ for nuclear counterstaining, mounted with $95 \%$ glycerol (Sigma-Aldrich), and observed by fluorescence microscope (Olympus IX71). Five random fields were selected and positive cells were counted. 

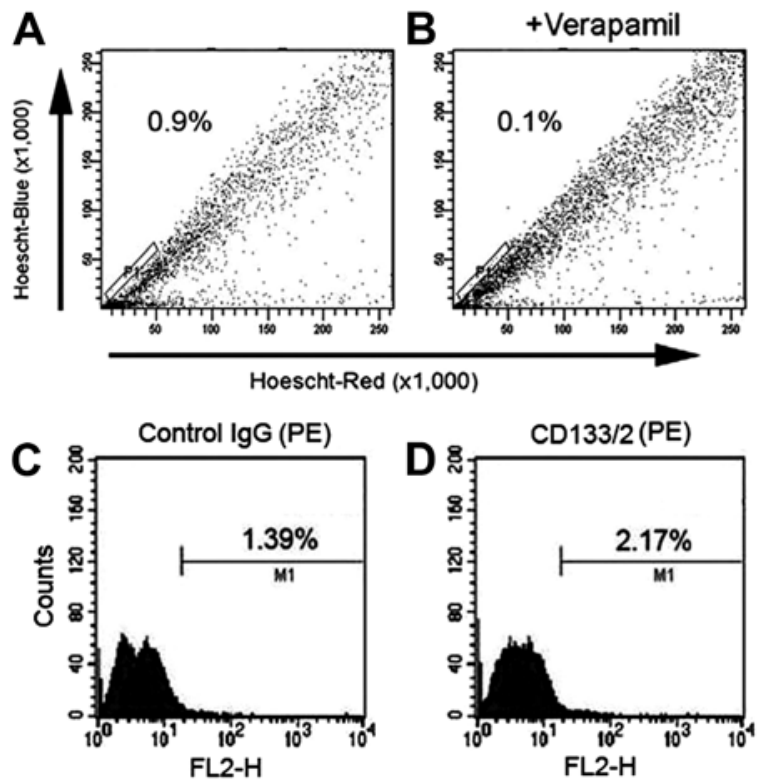

Figure 1. Ratio of CSCs isolated from SH-SY5Y cells analyzed by FACS. (A and B) Results of SP method. (A) Ratio of SP cells; (B) Ratio after verapamil treatment. (C and D) Results of surface marker method. (C) Ratio of control IgG group; (D) Ratio of CD133 antibody group.

Migration assay. To evaluate migratory properties of NB CSCs treated with XAV939 or RNAi, migration assay was used. Cells $\left(1 \times 10^{5}\right)$ were suspended in $100 \mu 1$ culture medium without FBS and loaded onto the top of Transwell chambers (24-well plate) equipped with $8.0 \mu \mathrm{m}$ pore-size polycarbonate membranes (Corning). Culture medium supplemented with 10\% FBS was used as chemotactic stimuli in the bottom chambers. After $24 \mathrm{~h}$ of incubation, cells on the upper surface of the filter were mechanically removed with a cotton swab, and those which migrated underneath the surface were fixed with $90 \%$ ethanol and stained with $0.1 \%$ crystal violet dye. Photographs were taken using an inverted phase contrast microscope (Olympus CK40) and the number of cells was counted.

Statistical analysis. The results obtained from three independent experiments performed in triplicate are presented as mean \pm SD. One-way ANOVA was performed for comparison between each group. P-values of $<0.05$ were considered as statistically significant.

\section{Results}

The comparison of two methods. The SP and non-SP (NSP) cells were sorted from the SH-SY5Y cell line. The P1 gate containing the SP cells accounted for $0.9 \pm 0.06 \%$ of the total cells (Fig. 1A). The percentage of SP cells decreased to $0.1 \%$ after treatment with verapamil, indicating that this population consisted of SP cells (Fig. 1B). However, the SP cell population is not obvious in flow chart and with poor specificity. Moreover, Hoechst 33342 is harmful to cells. So the SP cells after sorting had high death rate and poor culture result. SH-SY5Y cells were also analyzed by FACS using the characterization of CD133 expression on NB CSCs surface. The results showed that the ratio of control $\mathrm{IgG}$ group was $1.39 \%$ (Fig. 1C), while that of CD133 antibody group was $2.17 \%$ (Fig. 1D). Therefore the ratio of $\mathrm{CD}_{133}{ }^{+}$cells in SH-SY5Y cell line was $0.78 \%$.

Enriched NB CSCs by etoposide. CD133 are commonly used as cell surface markers representing the CSC subpopulation in NB (18). CSCs are resistant to conventional chemotherapy, and therefore we further investigate the enrichment of NB CSCs in SH-SY5Y cells using different concentrations of etoposide and sorted NB CSCs with the cell surface marker of CD133 (Fig. 2). After treatment with etoposide of 20, 40 and $60 \mu \mathrm{M}$,
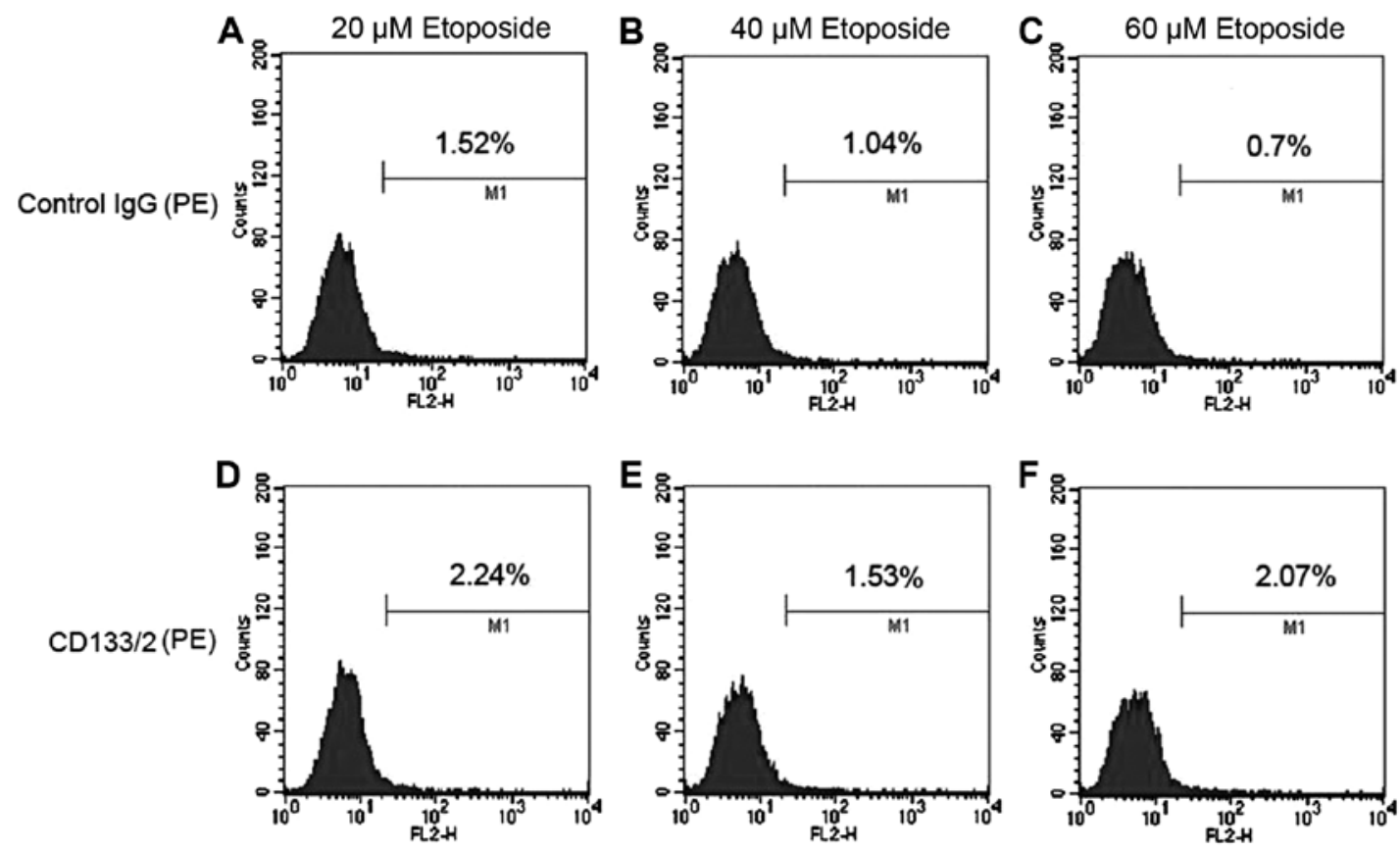

Figure 2. Ratios of CSCs isolated from SH-SY5Y cells treated with etoposide of different concentrations. (A-C) Ratios of control IgG groups; (D-F) Ratios of CD133/2 antibody groups. (A and D), (B and E) and (C and F) display the results of 20, 40 and $60 \mu \mathrm{M}$ etoposide groups, respectively. 


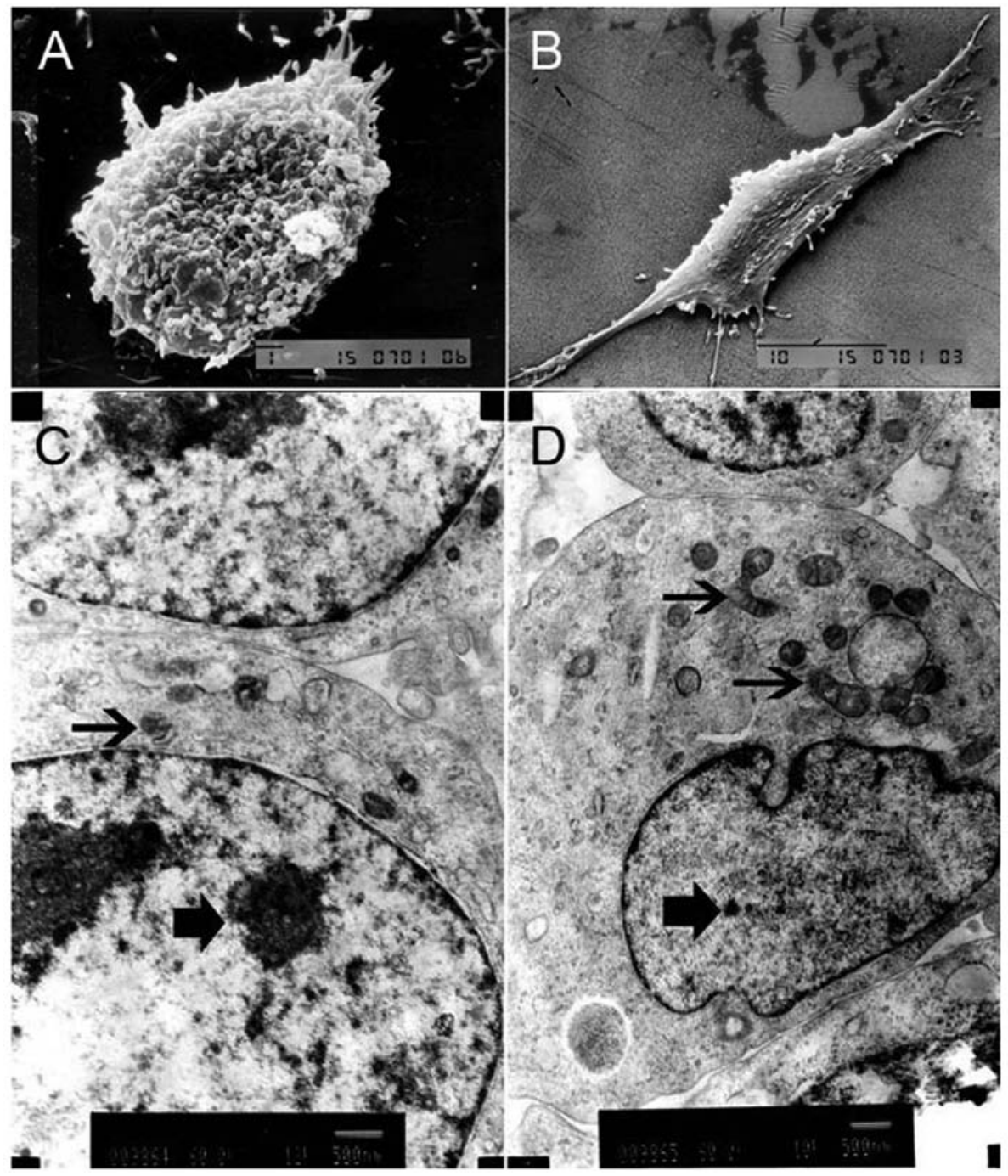

Figure 3. Ultrastructure of CSCs and non-CSCs (A and B) were observed by scanning electron microscopy. (A) The structure feature on CSCs on the surface, (B) non-CSCs. (C and D) were observed by transmission electron microscopy. (C) The internal structure of CSCs, (D) non-CSCS. The thin arrows point at organelles and the bold arrow points at the nucleolus. (A) Bar, $1 \mu \mathrm{m}$. (B) Bar, $10 \mu \mathrm{m}$. (C and D) Bar, $500 \mathrm{~nm}$.

the flow cytometry analysis showed that the ratio of NB CSCs was $0.72,0.49$ and $1.37 \%$, respectively. The ratio of NB CSCs in $60 \mu \mathrm{M}$ etoposide treatment group increased to nearly 2-fold of non-treatment group (Figs. 1 and 2), and was the highest among all groups.

The ultrastructure of $N B C S C s$. Under a SEM, NB CSCs were rounded or oval with rich and trivial cell processes on the surface, which gathered into a ball growth, indicating that it was in an undifferentiated state (Fig. 3A). However, the non-NB CSCs were elongated and spindle-like with poor but obvious cell processes (Fig. 3B). TEM showed the nuclear-cytoplasmic ratio of NB CSCs was higher than that of non-NB CSCs, and the NB CSCs had fewer cytoplasmic organelles than the non-NB CSCs (Fig. 3C and D). The undeveloped cytoplasmic organelles, such as rough endoplasmic reticulum or Golgi apparatus, indicated that the NB CSCs were in immature or juvenile stage.

XAV939 treatment or RNAi-TNKS1 inhibits the expression of CD133 mRNA and protein. QRT-PCR and western blot anal- ysis were used respectively to validate the stemness change of NB CSCs after XAV939 treatment or RNAi-TNKS1. The results of $\mathrm{qRT}$-PCR showed that the melting curve of $\beta$-actin and CD133 was a standard single peak, indicating specific amplification products (Fig. 4A). After XAV939 treatment or RNAi-TNKS1, the relative expression of CD133 mRNA was $0.57 \pm 0.09$ and $0.52 \pm 0.11$, respectively, which were both lower than those in control and SCR group (Fig. 4B, P<0.05). The relative expression of CD133 protein was significantly lower in XAV939 treatment group and RNAi-TNKS1 group compared with control groups (Fig. 4C and $\mathrm{D}, \mathrm{P}<0.05$ ). The results indicated that both XAV939 treatment and RNAi-TNKS1 repressed the stemness of CSCs to some extent.

XAV939 treatment and RNAi-TNKS1 reduce the subcellular localization of CD133 protein. The immunofluorescence results showed that CD133 protein was expressed in the cytoplasm and membrane of NB CSCs, and presented red fluorescence, while the nucleus of cells presented blue fluorescence (Fig. 5A). After XAV939 treatment or RNAi-TNKS1, the number of NB CSCs reduced and some signs of apoptosis 

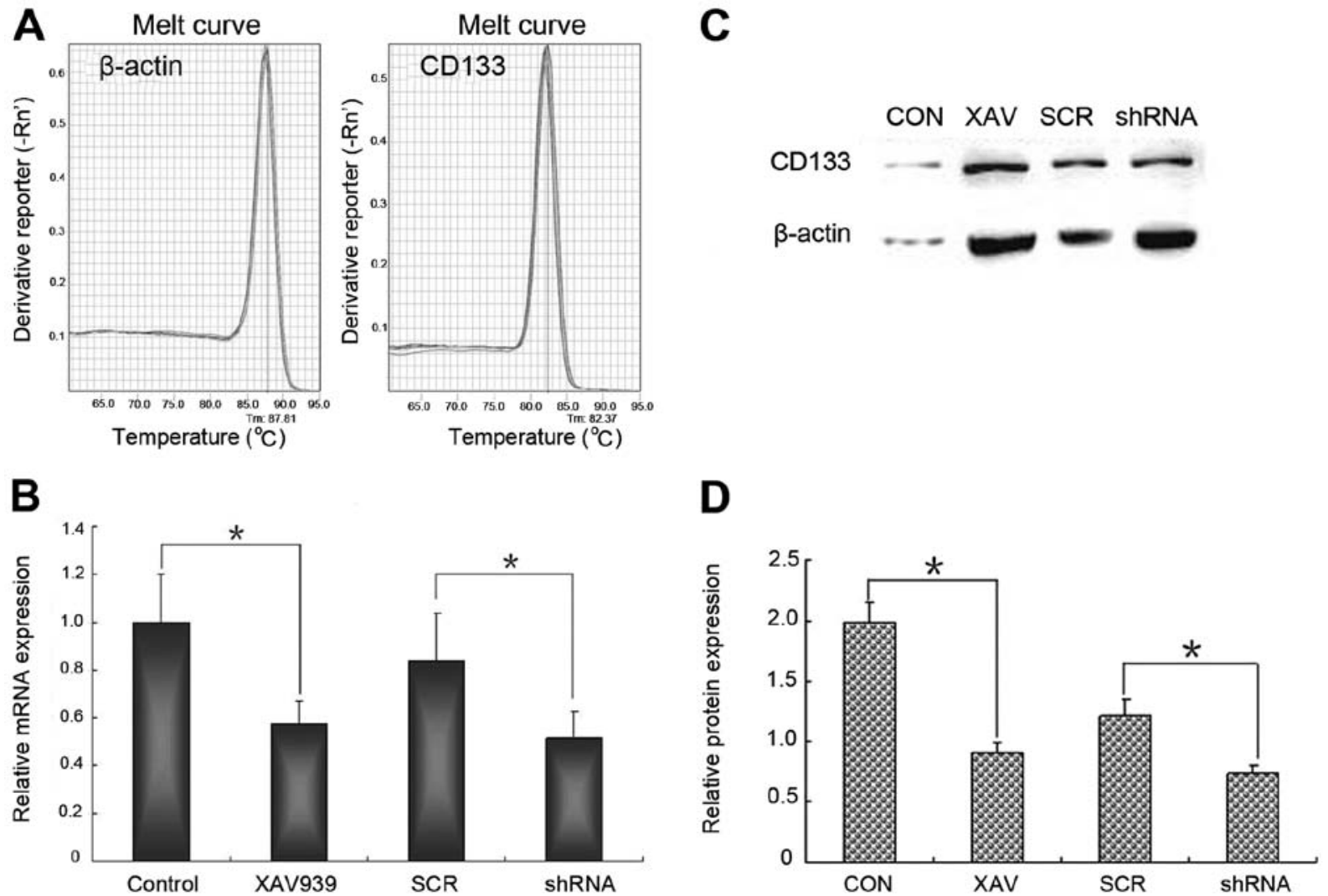

Figure 4. Expression of CD133 mRNA and protein. (A and B) Expression of CD133 mRNA detected by qRT-PCR. (A) Melting curves of $\beta$-actin and CD133; (B) Histogram of the relative expression of CD133 mRNA. (C and D) Expression of CD133 protein detected by western blot analysis. (C) Electrophoresis figure; (D) Histogram of the relative expression of $\mathrm{CD} 133$ protein. ${ }^{*} \mathrm{P}<0.05$.

appeared, including the condensation and bright stained of nuclear chromatin. The fluorescence intensity were analyzed with image software and presented in a histogram (Fig. 5B). The mean fluorescence intensity of CD133 protein in control, XAV939 treatment, SCR and shRNA group was $1 \pm 0.10$, $0.21 \pm 0.06,0.97 \pm 0.09$ and $0.41 \pm 0.08$, respectively. Compared with the respective control groups, XAV939 treatment and RNAi-TNKS1 significantly decreased the expression of CD133 protein $(\mathrm{P}<0.01)$. The change of mean fluorescence intensity of Hoechst 33342 showed similar tendency to that of CD133. The results indicated that XAV939 treatment and RNAi-TNKS1 reduced the expression of stemness of NB CSCs.

XAV939 treatment and RNAi-TNKS1 reduce the migration of $N B C S C$ s. In this study, 9 high power field of view were selected randomly, and cells migrated to the bottom of the wells were counted. As shown in Fig. 6A, the mean number of the migrated cells in control, XAV939 treatment, SCR and shRNA group was $62.0 \pm 6.0,36.7 \pm 4.5,63.1 \pm 2.0$ and $40.1 \pm 1.9$, respectively (Fig. 6B, $\mathrm{P}<0.05$ ). The results demonstrated that XAV939 treatment and RNAi-TNKS1 both decreased the migration of NB CSCs.

\section{Discussion}

CSCs may play an important role in curing cancer because CSCs have special biological characteristics, such as self- renewal, drug resistance and tumor forming (19-22). Traditional treatments can not effectively kill CSCs, and tumor will recur after treatment. Therefore, the study of CSCs will provide a new theoretical basis for curing cancer. The isolating of CSCs is the basis and difficulty for further research because of its very small proportion in cell lines or tumor tissues, which accounts for $0.01-2 \%$ of the total number of cells. There are some methods for isolating CSCs at present, such as SP method, surface marker method, and floating sphere formation method (23-29). It has also been reported that SH-SY5Y cells expressed CD133, but did not form tumor spheres (30), which was consistent with the result of our preliminary experiments (data not shown). Therefore, we compared the advantage and disadvantage of SP method and surface marker method for isolating NB CSCs. The results demonstrated that SP method had universal applicability and poor specificity, which was consistent with a report of that not all SP cells had the characteristic of CSCs (31). Besides, the fluorescent dye Hoechst 33342 could bind with DNA through the membrane and had toxic effects. Thus SP method is suitable for cells without significant surface markers. In contrast, surface marker method had higher specificity. Studies showed that tumor spheres obtained from solid tumor or cell lines were positively stained with CD133, and CD133 was considered to be the most reliable marker of brain CSCs by far (32). Our study used the cell surface marker CD133 method for isolating NB CSCs also in followup studies. 
A
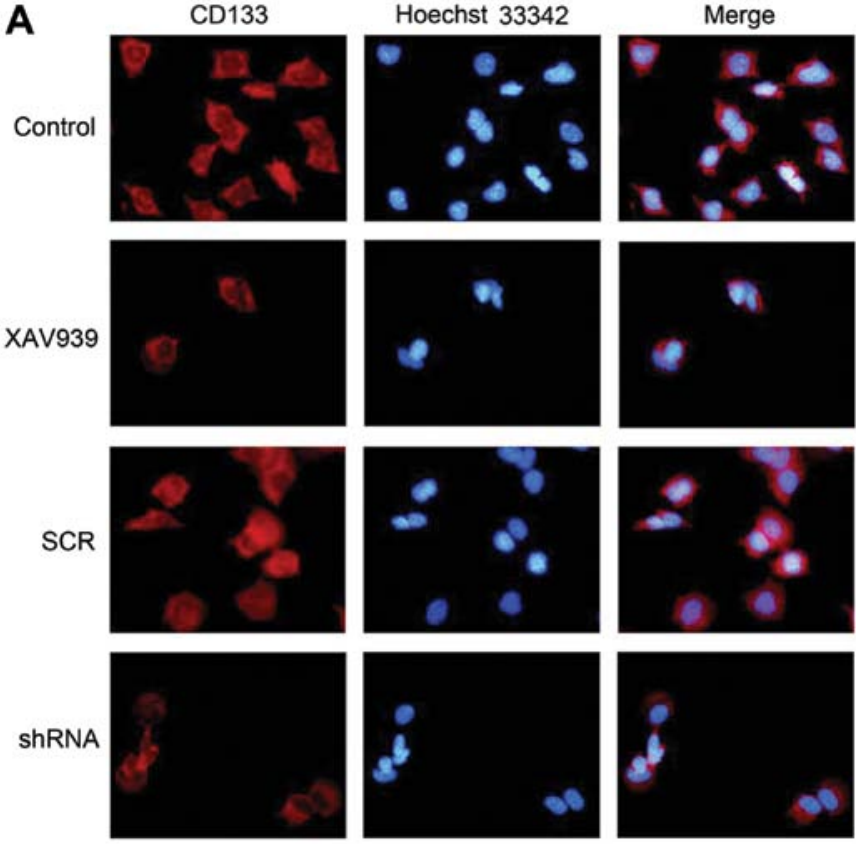

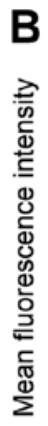

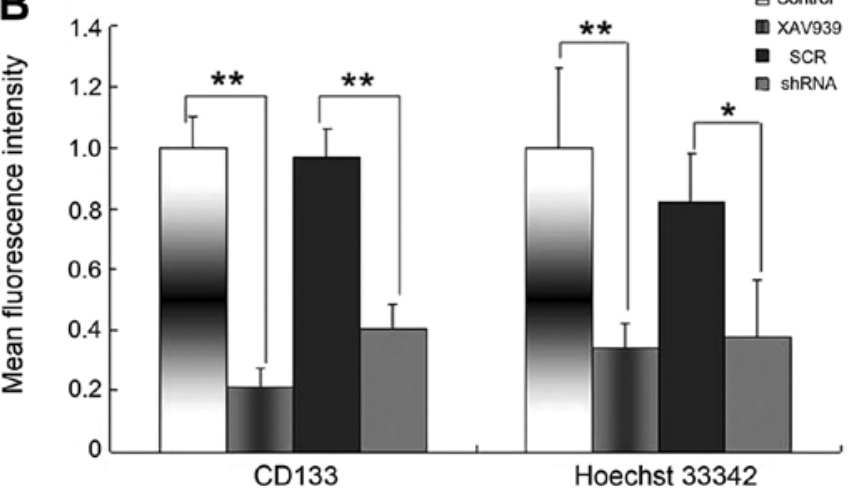

Figure 5. Subcellular localization and expression of CD133 protein after XAV939 treatment and RNAi-TNKS1 in CSCs. (A) Immunofluorescence results. The red fluorescence represents CD133 protein, blue fluorescence represents the nucleus and the merge represents overlapping of the two. (B) Mean fluorescence intensity of CD133 protein and Hoechst 33342 in control, XAV939 treatment, SCR and shRNA groups. ${ }^{*} \mathrm{P}<0.05,{ }^{* *} \mathrm{P}<0.01$.

The NB CSCs usually are enriched first because their small proportion in SH-SY5Y cells. There are some methods for the enrichment of CSCs, such as culturing in serum-free medium (SFM) with specific growth factors and cytokines, chemotherapy drug screening and long-term hypoxia (1\% $\mathrm{O}_{2}, 30$ d) intervention (30,33-35). Since SH-SY5Y cells do not have the ability to form tumor spheres (30) and hypoxia method takes more time, we used etoposide treatment to enrich NB CSCs. Then isolated NB CSCs were cultured with SFM to maintain the stemness properties. The method is simple and low cost, and the ratio of NB CSCs increased to nearly 2 -fold. The isolated NB CSCs were further identified with an electron microspe, and the results indicated that NB CSCs were in immature or juvenile stage, which was in line with the characteristics of stem cells (Fig. 3).

CD133, also known as AC133, is encoded by the PROMI gene. A notable feature of $\mathrm{CD} 133$ is that its expression will
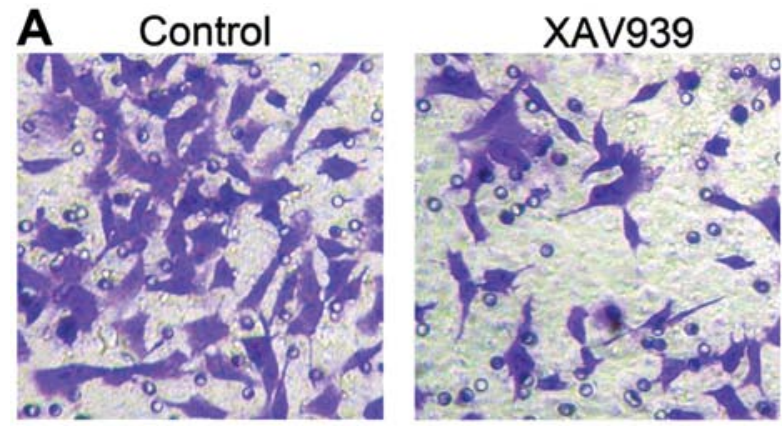

SCR

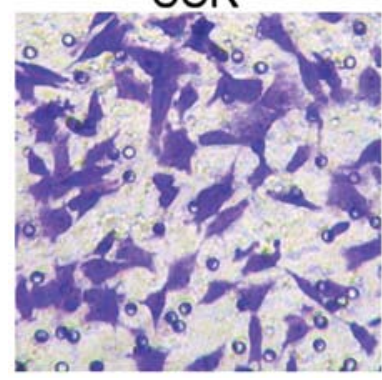

B

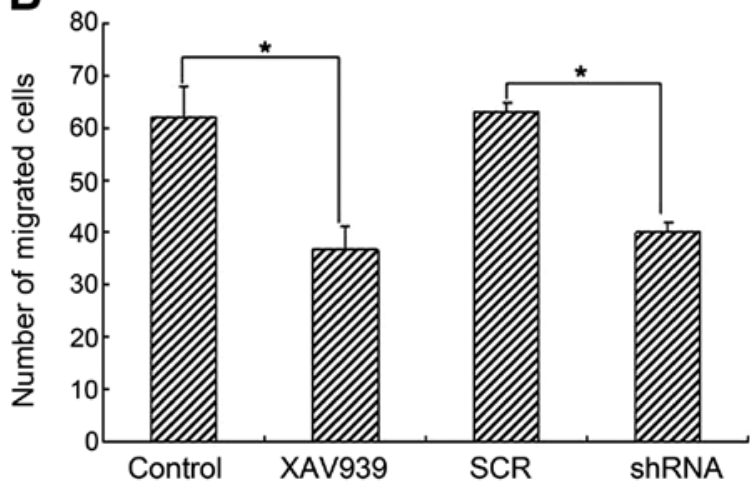

Figure 6. Migration of CSCs after XAV939 treatment and RNAi-TNKS1. (A) CSCs migrated to the bottom of the wells which were stained with crystal violet. (B) Histogram of migrated CSCs in different groups. ${ }^{*} \mathrm{P}<0.05$.

downregulate quickly with cell differentiation (29), and make it a unique molecular marker for isolation and identification of NB CSCs $(28,29)$. It has been reported that the higher the degree of malignancy, the higher the proportion of $\mathrm{CD} 133^{+}$ tumor cells are accounted for (36). In our previous studies, we have demonstrated that XAV939 promoted cell apoptosis in NB cell lines in part by inhibiting the Wnt/ $\beta$-catenin signaling pathway (17). Since CSCs have similar signaling pathways with normal stem cells, such as Notch, and Wnt, which are closely related to the self-renewal ability or stemness of CSCs (37), studies have also shown that abnormal activation of the Wnt signaling pathway can induce the transformation from stem cells to tumor cells (38), and this signaling pathway plays an important role in maintaining the characteristics of CSCs (39-42). Kim et al found that the increased expression of Wnt inhibitory factor promoted the apoptosis of tumor cells, reduced colony formation rate and significantly inhibited tumor growth (43). The encoding gene of CD133 contains TCF/LEF binding region, which suggested that Wnt/ $\beta$-catenin signaling pathway might associate with the 
expression of CD133 (44) and CD133 might be a downstream target gene of this pathway (45). Therefore, we speculated that XAV939 might inhibit the stemness of NB CSCs by attenuating Wnt/ $\beta$-catenin pathway and expression of CD133 via repression of TNKS1. Targeting the key genes conferring stemness to NB CSCs can efficiently eliminate NB CSCs, and thus may be considered to provide a new approach to cancer therapy. In our research, the stemness and migration ability were inhibited markedly by the repression of TNKS1 gene. It is foreseeable that the combination of multiple targets as a potential anticancer project will be further studied in this direction (46).

Upon treatment with XAV939, we observed a significant reduction in the NB CSCs marker expression. In addition, when we knocked down the TNKS1 gene, the subpopulation and migration of NB CSCs were reduced, suggesting a pivotal role of TNKS1 in the maintainence and movement of NB CSCs. Moreover, our present and previous studies showed that inhibited TNKS1 was correlated with increased apoptosis in NB cells, increased chemosensitivity and reduced invasiveness. Although TNKS1 may be necessary in kidney and lung development, its overexpression in many kinds of cancers is notable, thus make it an attractive target for certain cancer therapies $(47,48)$.

In conclusion, we found that surface marker CD133 was more suitable than SP method for isolating CSCs from NB cell line, and $60 \mu \mathrm{M}$ etoposide could be used to enrich NB CSCs. The ultrastructure of CSCs showed its juvenescence or stemness state. The small molecule drug XAV939 or RNAi-TNKS1 appeared to inhibit the stemness and migration of NB CSCs via repression of TNKS1 at different aspects evidenced by the decreased expression of NB CSCs marker CD133 and decreased migration. The findings in the present study provide a basis for the multiple-targeted therapy of NB CSCs by small molecule drugs, and need to be verified in vivo.

\section{Acknowledgements}

This study was supported by National Natural Science Foundation of China (30772215). The authors are grateful to the Experimental Technology Center, Department of Developmental Biology, Department of Pharmacology and Department of Pathophysiology in China Medical University. We deeply thank all the people who helped us.

\section{References}

1. Hamburger AW and Salmon SE: Primary bioassay of human tumor stem cells. Science 197: 461-463, 1977.

2. Reya T, Morrison SJ, Clarke MF and Weissman IL: Stem cells, cancer, and cancer stem cells. Nature 414: 105-111, 2001.

3. Huntly BJP and Gilliland DG: Leukaemia stem cells and the evolution of cancer-stem-cell research. Nat Rev Cancer 5: 311-321, 2005

4. Ross RA and Spengler BA: Human neuroblastoma stem cells. Semin Cancer Biol 17: 241-247, 2007.

5. Bilir A, Erguven M, Yazihan N, Aktas E, Oktem G and Sabanci A: Enhancement of vinorelbine-induced cytotoxicity and apoptosis by clomipramine and lithium chloride in human neuroblastoma cancer cell line SH-SY5Y. J Neurooncol 100 : 385-395, 2010.

6. Abbott BL: ABCG2 (BCRP): a cytoprotectant in normal and malignant stem cells. Clin Adv Hematol Oncol 4: 63-72, 2006.
7. Nan JN, Hu XG and Li HX: Research progress in tumor stem cells: literature retrieval results based on international database. Chin J Tiss Eng Res 516: 1085-1093, 2012.

8. Huang SM, Mishina YM, Liu S, et al: Tankyrase inhibition stabilizes axin and antagonizes Wnt signaling. Nature 461: 614-620, 2009.

9. Chen B, Dodge ME, Tang W, et al: Small molecule-mediated disruption of Wnt-dependent signaling in tissue regeneration and cancer. Nat Chem Biol 5: 100-107, 2009.

10. Xu D, Zheng C, Bergenbrant S, Holm G, Björkholm M, Yi Q and Gruber A: Telomerase activity in plasma cell dyscrasias. $\mathrm{Br}$ J Cancer 84: 621-625, 2001.

11. MacNamara B, Wang W, Chen Z, et al: Telomerase activity in relation to pro- and anti-apoptotic protein expression in high grade non-Hodgkin's lymphomas. Haematologica 86: 386-393, 2001.

12. Klapper W, Krams M, Qian W, Janssen D and Parwaresch R: Telomerase activity in B-cell non-Hodgkin lymphomas is regulated by hTERT transcription and correlated with telomerebinding protein expression but uncoupled from proliferation. $\mathrm{Br}$ J Cancer 89: 713-719, 2003.

13. Gelmini S, Poggesi M, Distante V, et al: Tankyrase, a positive regulator of telomere elongation, is over expressed in human breast cancer. Cancer Lett 216: 81-87, 2004.

14. Gelmini S, Poggesi M,Pinzani P, et al: Distribution of tankyrase-1 mRNA expression in colon cancer and its prospective correlation with progression stage. Oncol Rep 16: 1261-1266, 2006.

15. Gelmini S, Quattrone S, Malentacchi F, et al: Tankyrase-1 mRNA expression in bladder cancer and paired urine sediment: preliminary experience. Clin Chem Lab Med 45: 862-866, 2007.

16. Shervington A, Patel R, Lu C, et al: Telomerase subunits expression variation between biopsy samples and cell lines derived from malignant glioma. Brain Res 1134: 45-52, 2007.

17. Tian XH, Hou WJ, Fang Y, et al: XAV939, a tankyrase 1 inhibitior, promotes cell apoptosis in neuroblastoma cell lines by inhibiting Wnt/ $\beta$-catenin signaling pathway. J Exp Clin Cancer Res 32: 100-109, 2013.

18. Vangipuram SD, Buck SA and Lyman WD: Wnt pathway activity confers chemoresistance to cancer stem-like cells in a neuroblastoma cell line. Tumour Biol 33: 2173-2183, 2012.

19. Al-Hajj M and Clarke MF: Self renewal and solid tumor stem cells. Oncogene 23: 7274-7282, 2004.

20. Dean M, Fojo T and Bates S: Tumour stem cells and drug resistance. Nat Rev Cancer 5: 275-284, 2005.

21. Lou H and Dean M: Targeted therapy for cancer stem cells: the patched pathway and ABC transporters. Oncogene 26: 1357-1360, 2007.

22. Hadnagy A, Gaboury L, Beaulieu R and Balicki D: SP analysis may be used to identify cancer stem cell populations. Exp Cell Res 312: 3701-3710, 2006.

23. Al-Hajj M, Wicha MS, Benito-Hernandez A, Morrison SJ and Clarke MF: Prospective identification of tumorigenic breast cancer cells. Proc Natl Acad Sci USA 100: 3983-3988, 2003.

24. Bonnet D and Dick JE: Human acute myeloid leukemia is organized as a hierarchy that originates from a primitive hematopoietic cell. Nat Med 3: 730-737, 1997.

25. Collins AT, Berry PA, Hyde C, Stower MJ and Maitland NJ: Prospective identification of tumorigenic prostate cancer stem cells. Cancer Res 65: 10946-10951, 2005.

26. O'Brien CA, Pollett A, Gallinger S and Dick JE: A human colon cancer cell capable of initiating tumour growth in immunodeficient mice. Nature 445: 106-110, 2007.

27. Ricci-Vitiani L, Lombardi DG, Pilozzi E, et al: Identification and expansion of human colon-cancer-initiating cells. Nature 445: 111-115, 2007.

28. Singh SK, Clarke ID, Terasaki M, et al: Identification of a cancer stem cell in human brain tumors. Cancer Res 63: 5821-5828, 2003.

29. Singh SK, Hawkins C, Clarke ID, et al: Identification of human brain tumor initiating cells. Nature 432: 396-401, 2004.

30. Mahller YY, Williams JP, Baird WH, et al: Neuroblastoma cell lines contain pluripotent tumor initiating cells that are susceptible to a targeted oncolytic virus. PLoS One 4: e4235, 2009.

31. Hemmati HD, Nakano I, Lazareff JA, et al: Cancerous stem cells can arise from pediatric brain tumors. Proc Natl Acad Sci USA 100: 15178-15183, 2003

32. Na YR, Seok SH, Kim DJ, et al: Isolation and characterization of spheroid cells from human malignannt melanoma cell line WM-266-4. Tumor Biol 30: 300-309, 2009. 
33. Di Fiore R, Santulli A, Ferrante RD, et al: Identification and expansion of human osteosarcoma-cancer-stem cells by long-term 3-aminobenzamide treatment. J Cell Physiol 219: 301-313, 2009.

34. Das B, Tsuchida R, Malkin D, Koren G, Baruchel S and Yeger H: Hypoxia enhances tumor stemness by increasing the invasive and tumorigenic side population fraction. Stem Cells 26: 1818-1830, 2008.

35. Marzi I, D'Amico M, Biagiotti T, et al: Purging of the neuroblastoma stem cell compartment and tumor regression on exposure to hypoxia or cytotoxic treatment. Cancer Res 67: 2402-2407, 2007.

36. Deng YW, Fang JS, Li MC, et al: Correlation research between cancer stem cells and the pathological grades of neuroepithelial tumors. J Cent South Uniu (Med Sci) 31: 45-51, 2006.

37. Abbott A: Cancer: the root of the problem. Nature 442: 742-743, 2006.

38. Lindvall $\mathrm{C}, \mathrm{Bu} \mathrm{W}, \mathrm{Williams} \mathrm{BO}$ and $\mathrm{Li} \mathrm{Y}$ : Wnt signaling, stem cells, and the cellular origin of breast cancer. Stem Cell Rev 3: 157-168, 2007.

39. Fu JK, Wang Z and Wei S: Alterations and its mechanisms of Wnt signal pathway in human high-matastatatic large cell lung cancer cell line L9981 by transfecting with Nm23-H1 gene. Chin J Lung Cancer 12: 477-479, 2009.

40. Nguyen DX, Chiang AC, Zhang XH, et al: WNT/TCF signaling through LEF1 and HOXB9 mediates lung adenocarcinoma metastasis. Cell 138: 51-62, 2009.

41. Akiri G, Cherian MM, Vijayakumar S, Liu G, Bafico A and Aaronson SA: Wnt pathway aberrations including autocrine Wnt activation occur at high frequency in human non-small-cell lung carcinoma. Oncogene 28: 2163-2172, 2009.
42. Sun JZ, Yang XX, Hu NY, Li X, Li FX and Li M: Genetic variants in MMP9 and TGF2 contribute to susceptibility to lung cancer. Chin J Cancer 23: 183-187, 2011.

43. Kim J, You L, Xu Z, et al: Wnt inhibitory factor inhibits lung cancer cell growth. J Thorac Cardiovasc Surg 133: 733-737, 2007.

44. Katoh Y and Katoh M: Comparative genomics on PROM1 gene encoding stem cell marker CD133. Int J Mol Med 19: 967-970, 2007.

45. Horst D, Kriegl L, Engel J, Jung A and Kirchner T: CD133 and nuclear beta-catenin: the marker combination to detect high risk cases of low stage colorectal cancer. Eur J Cancer 45: 2034-2040, 2009.

46. Ruck P, Xiao JC, Pietsch T, Von Schweinitz D and Kaiserling E: Hepatic stem-like cells in hepatoblastoma: expression of cytokeratin 7, albumin and oval cell associated antigens detected by OV-1 and OV-6. Histopathology 31: 324-329, 1997.

47. Karner CM, Merkel CE, Dodge M, Ma Z, Lu J, Chen C, Lum L and Carroll TJ: Tankyrase is necessary for canonical Wnt signaling during kidney development. Dev Dyn 239: 2014-2023, 2010.

48. Chung SS, Giehl N, Wu Y and Vadgama JV: STAT3 activation in HER2-overexpressing breast cancer promotes epithelialmesenchymal transition and cancer stem cell traits. Int J Oncol 44: 403-411, 2014 